\title{
Liste des Éphéméroptères-hôtes de parasites, de commensaux et autres associés
}

\author{
par L. ARVY * et W. L. PETERS * * \\ * Laboratoire Histoenzymologie, \\ Faculté de Médecine, 45, rue des Saints Pères, F 75006 Paris. \\ ** Laboratory of Aquatic Entomology, Florida A and M Univ., Tallahassee, FL 32307, E.U.A.
}

\section{Résumé.}

Pour la première fois, tous les associés, commensaux ou parasites des Ephéméroptères sont inventoriés.

\section{Sumimary.}

Ephemeroptera-host list for associated organisms.

For the first time an Ephemeroptera-host list is given for organisms found on, in or directly dependent upon Ephemeroptera.

Poursuivant nos recherches sur les êtres vivant en association, bénéfique ou mortelle, avec les éphéméroptères, nous avons jugé utile de résumer en les systématisant les données que nous avons assemblées en cinq années de recherches.

Depuis le début du $\mathrm{xx}^{\mathrm{e}}$ siècle, les acceptions ont varié quant aux appellations des partenaires: commensal, mutualiste, inquilin, parasite; la simple lecture des quelques ouvrages traitant de cette question (1 à 4), comme la lecture des conclusions des diver- 
ses commissions de terminologie, ne laisse pas parfaitement satisfait; il nous a donc paru utile de préciser nos conceptions.

Certains groupements sociaux impliquent une solidarité peu apparente, permanente ou plus ou moins temporaire, provoquée par une conjonction de divers facteurs indéterminés, parmi lesquels on peut accepter le choix d'un même biotope pour la ponte, des périodes d'éclosion contemporaines... etc. De tels groupements peuvent être saisonniers et plus ou moins localisés dans l'espace; il en existe deux exemples-types : les groupements de Rotifères au voisinage des larves d'Ephéméroptères, ou de larves d'Ephéméroptères au voisinage des Unionidés ; ces groupements locaux n'ont vraisemblablement pas d'importance vitale pour aucun des associés.

Un autre type d'association, le plus souvent dépourvu d'importance vitale pour les membres du groupe, comprend des cas de phorésie externe, l'un des associés se fixe sur l'autre qui le transporte, apparemment comme le ferait un support inerte. Théoriquement au moins, toute la faunule sessile, plus ou moins cosmopolite du biotope, peut se fixer sur des larves d'Ephéméroptères, fortuitement, ou par choix et de manière permanente. Exceptionnellement, l'un de nous (L.A.) a vu une larve d'Ecdyonurus transporter sur son dos une ponte (vraisemblablement d'un Tabanidé), probablement fixée là, sans dommage pour l'éphémère. Les Chlorella transportés par des Caenis sont entièrement passifs, de même probablement que les Vorticella transportés par des Baetis. Il est difficile de décider si ces épizoaires se fixent par hasard, ou s'ils trouvent sur certaines larves d'éphémères des conditions de localisation favorables, qui conditionnent la spécificité apparente de leurs associations; il est probable que les Vorticelliens, en particulier, vivraient aussi bien s'ils étaient fixés sur un autre support; il est néanmoins vraisemblable que les déplacements des éphémères, les microcourants créés par les battements branchiaux... assurent aux Invertébrés fixés et ainsi transportés par leur hôte, certains bénéfices: rencontre plus fréquente de proies, eau constamment renouvelée, plus oxygénée... etc.; on peut donc admettre qu'il s'agisse de commensalisme ; il ne s'agit pas de mutualisme, car l'éphémère ne retire de cette association aucun bénéfice.

Il en est tout autrement avec les larves d'Epoicocladius, réels compagnons des larves d'éphémères auxquelles elles s'amarrent; elles ingèrent les débris des proies de l'insecte, nettoient soies et branchies de toutes particules étrangères; éphémère et Epoicocladius se rendant de mutuels services : ce sont des mutualistes.

D'autres phorésies sont semi-internes, tels les Paramoebidium ou les Genistella de l'ampoule rectale de certains Ephéméroptères, ils trouvent chez ces derniers un support et un abri. Les rapports entre les deux associés sont uniquement spatiaux, sans incidence directe sur la physiologie de l'hôte.

La plupart des grégarines d'éphémérines sont des commensales internes, sans effet nocif ; elles vivent des aliments en voie de digestion dans l'intestin de leur hôte ; chez certains Insectes, les gamontes grégariniens intraépithéliaux sont assez nombreux pour provoquer des destructions étendues des cellules intestinales, où ils grandissent 
jusqu'au moment de leur chute dans la lumière, mais des invasions grégariniennes massives de ce type n'ont pas été signalées chez les éphéméroptères.

Le Sporozoaire Spiriopsis adipophila (Arvy et Delage, 1966) est également peu léthalisant, bien qu'il provoque la disparition totale du tissu adipeux des larves ; cependant, sur plusieurs générations, sa présence se manifesterait pas une diminution du nombre des œufs et un certain nanisme des éphémères [Delvaux (5)].

D'autres parasites trouvent dans les larves d'éphémères des conditions si favorables qu'ils se multiplient aux dépens des divers tissus, jusqu'à envahir tout l'insecte, épuiser ses réserves, bloquer l'évolution des gonades, de telle sorte que les larves parasitées sont castrées; il en est ainsi avec certaines Microsporidies.

Certains parasites envahissent le milieu intérieur de l'hôte, sans y trouver les conditions idéales pour leur développement et leur multiplication; il en est ainsi de certaines larves de Trématodes ou de Gordiacés; ils s'enkystent et persistent en vie ralentie, l'éphémère servant d'hôte intermédiaire pour le parasite, jusqu'à la rencontre avec son hôte définitif, qui lui permettra d'achever son cycle vital.

Les organismes qui provoquent la mort des éphémères sans vivre en symbiose avec eux sont des prédateurs; de nombreux insectes aquatiques ingèrent les œufs, ou les. larves, d'éphémères; certains poissons, certains oiseaux, certaines chauves-souris et d'autres vertébrés ingèrent les œufs et les éphémères à tous les stades ; on connaît des insectes terrestres qui dévorent les éphémères [par exemple, le Coléoptère élatéride observé par Demoulin (6) : Corymbites purpureus (Poda) achevant de dévorer un subimago d'Ephemerella ignita (Poda)]. Dans notre liste des associés des Ephéméroptères, nous n'avons situé que les mites prédateurs, car il n'est pas facile de décider si un Acare est un parasite ou un prédateur.

La distinction entre les différents types d'associations n'est pas sans ambiguïté ; pour bien comprendre les rapports qui unissent dans chaque cas les associés, il convient de se reporter aux recherches originales, déjà collationnées par Peters et Arvy (7 et 8). ou citées ici en référence $(5,9$ à 13$)$. 


\section{ABRÉVIATIONS DES TYPES DE RAPPORTS}

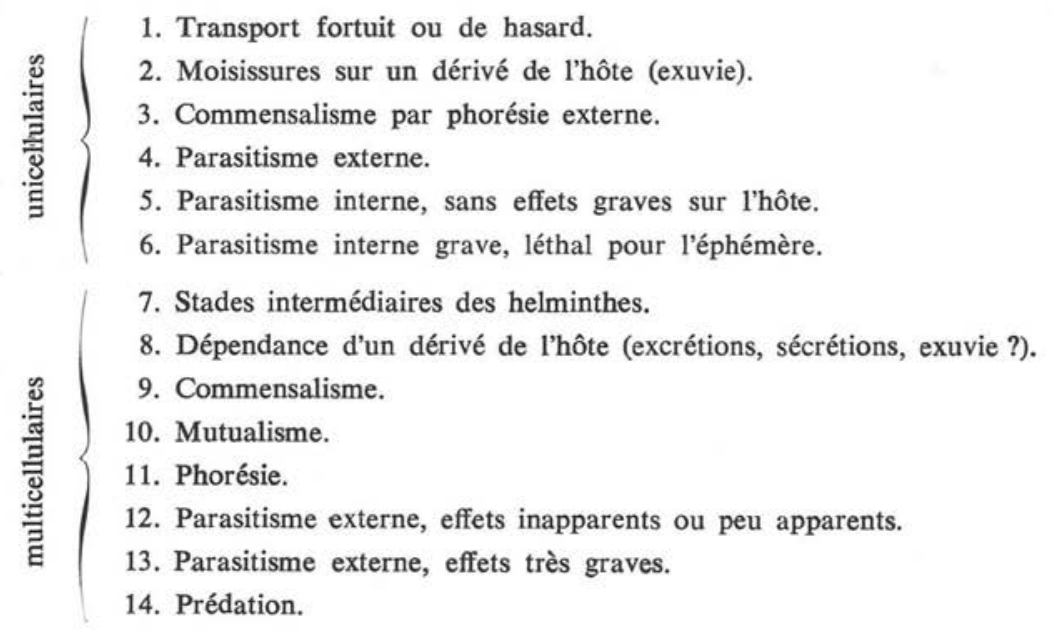

Abréviations DES ORganismes

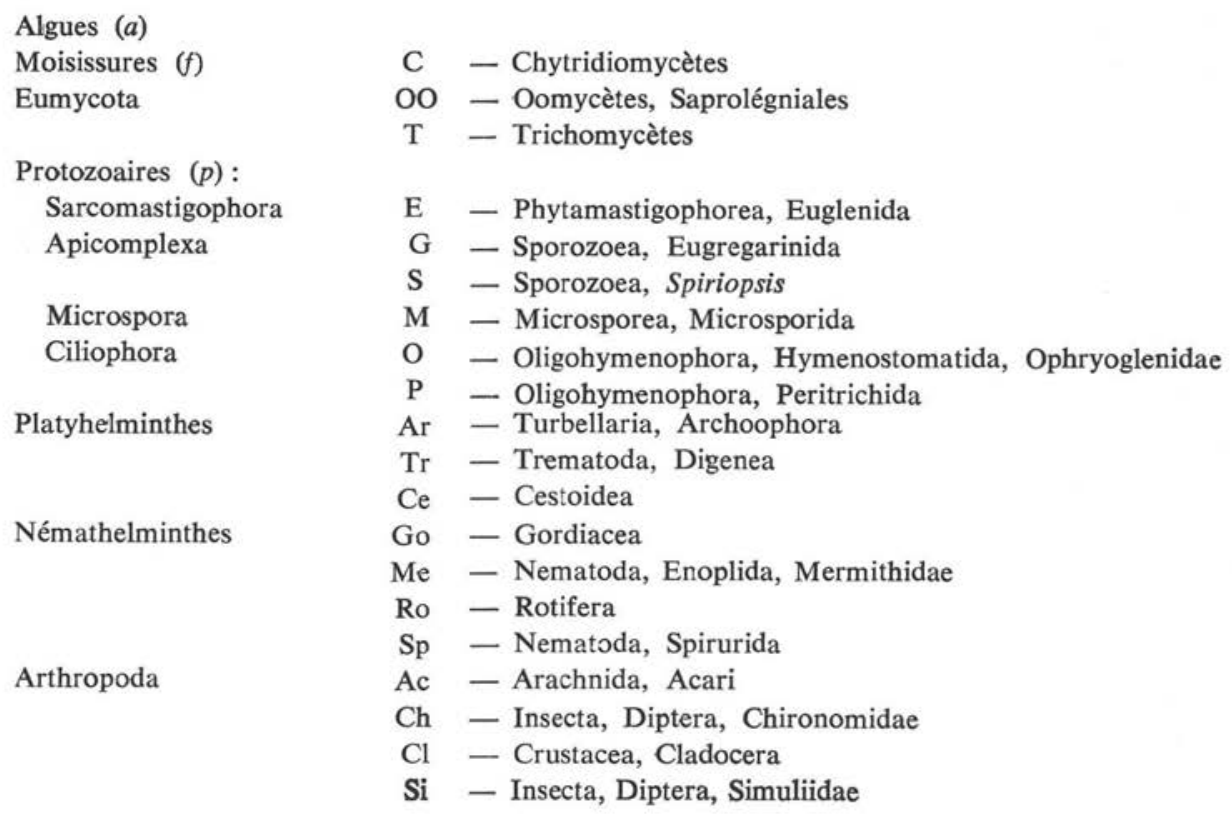




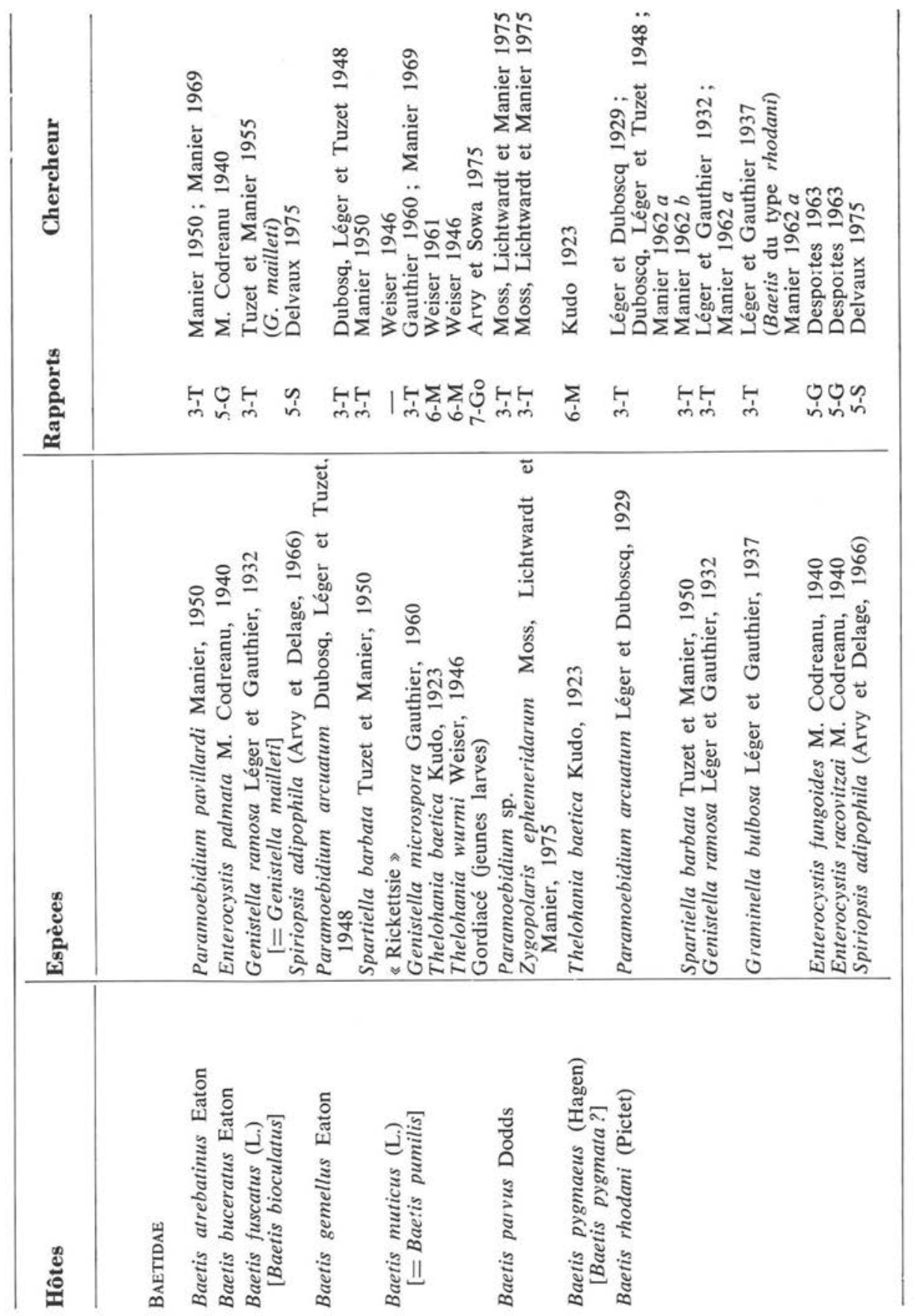




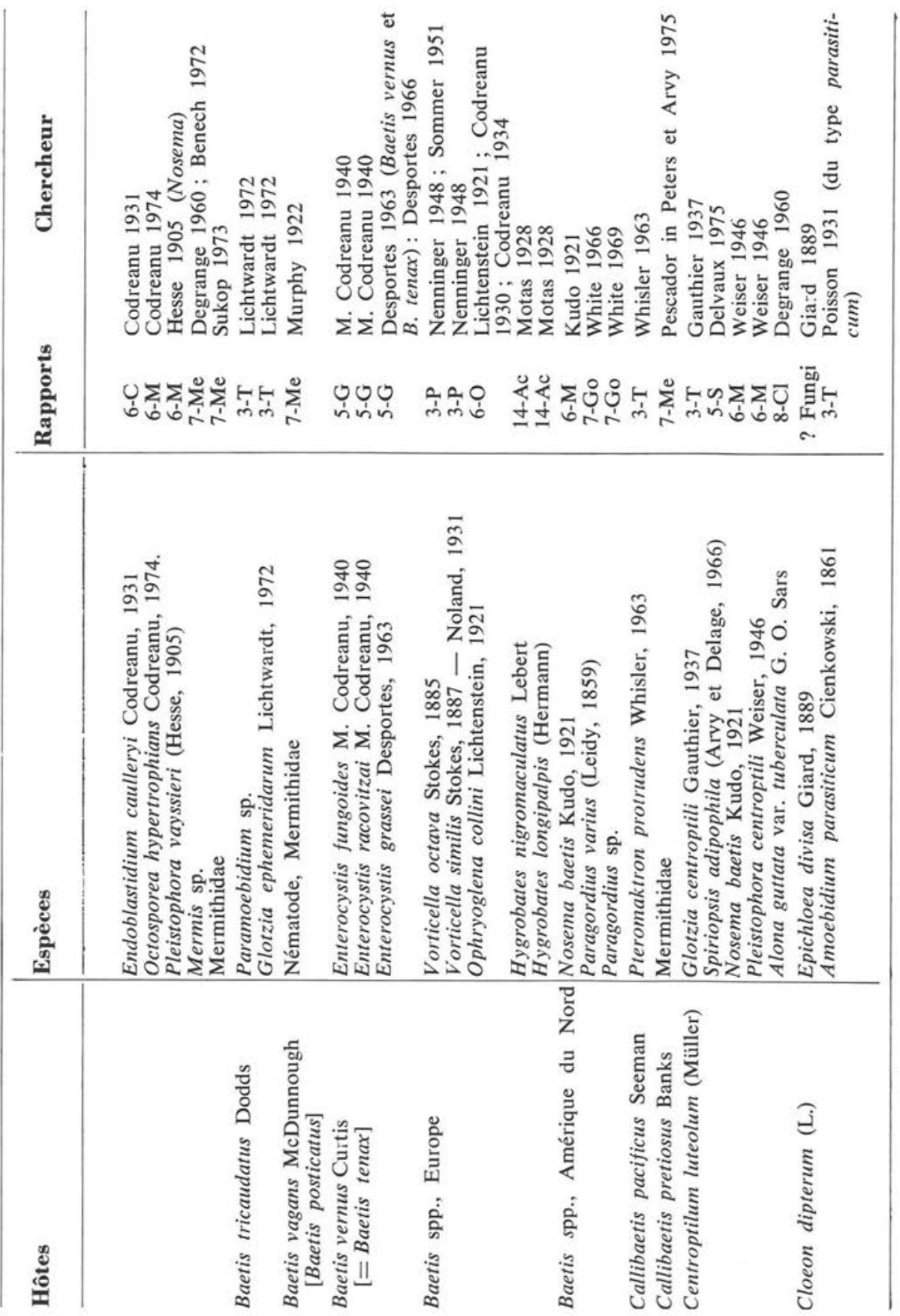




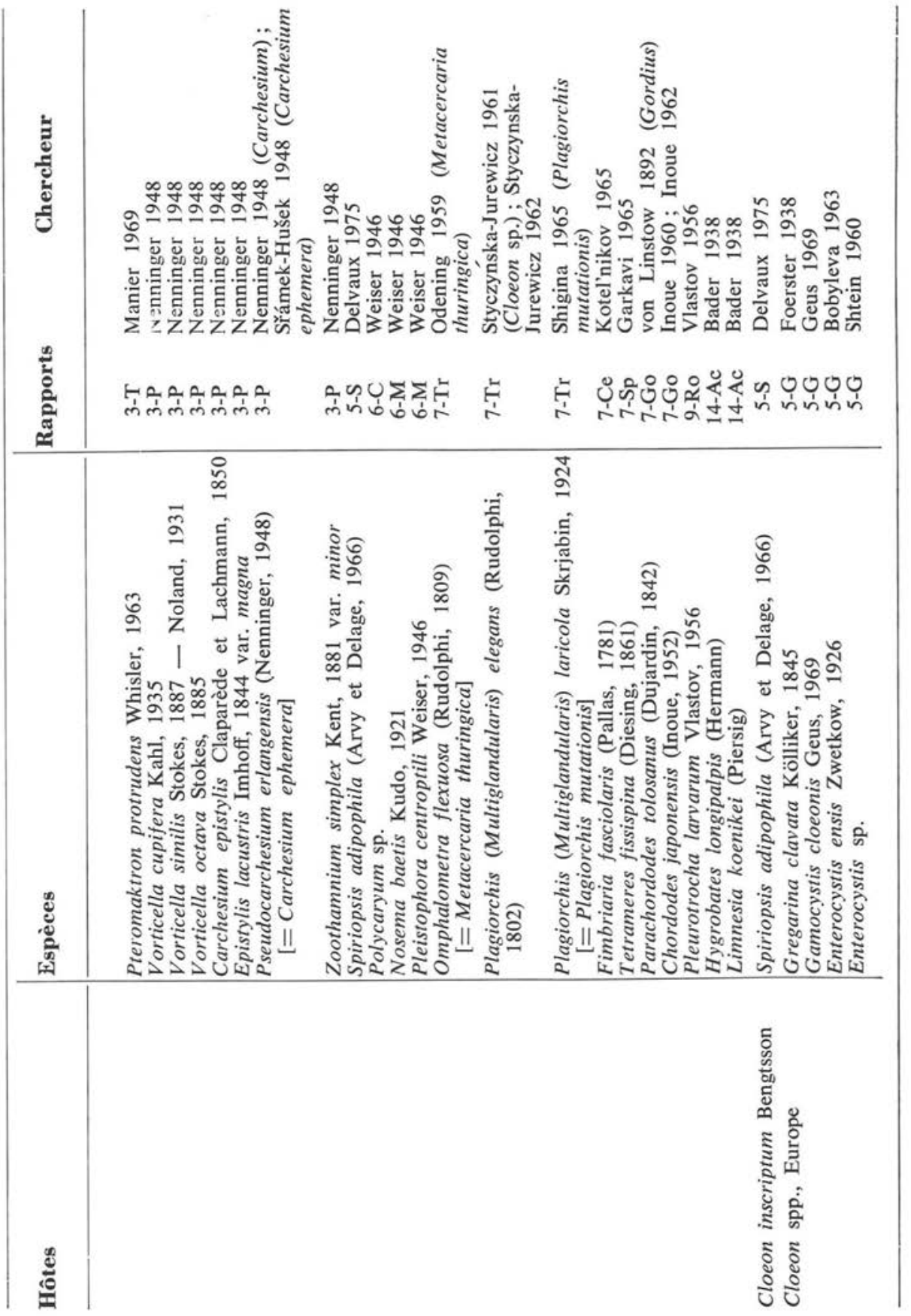




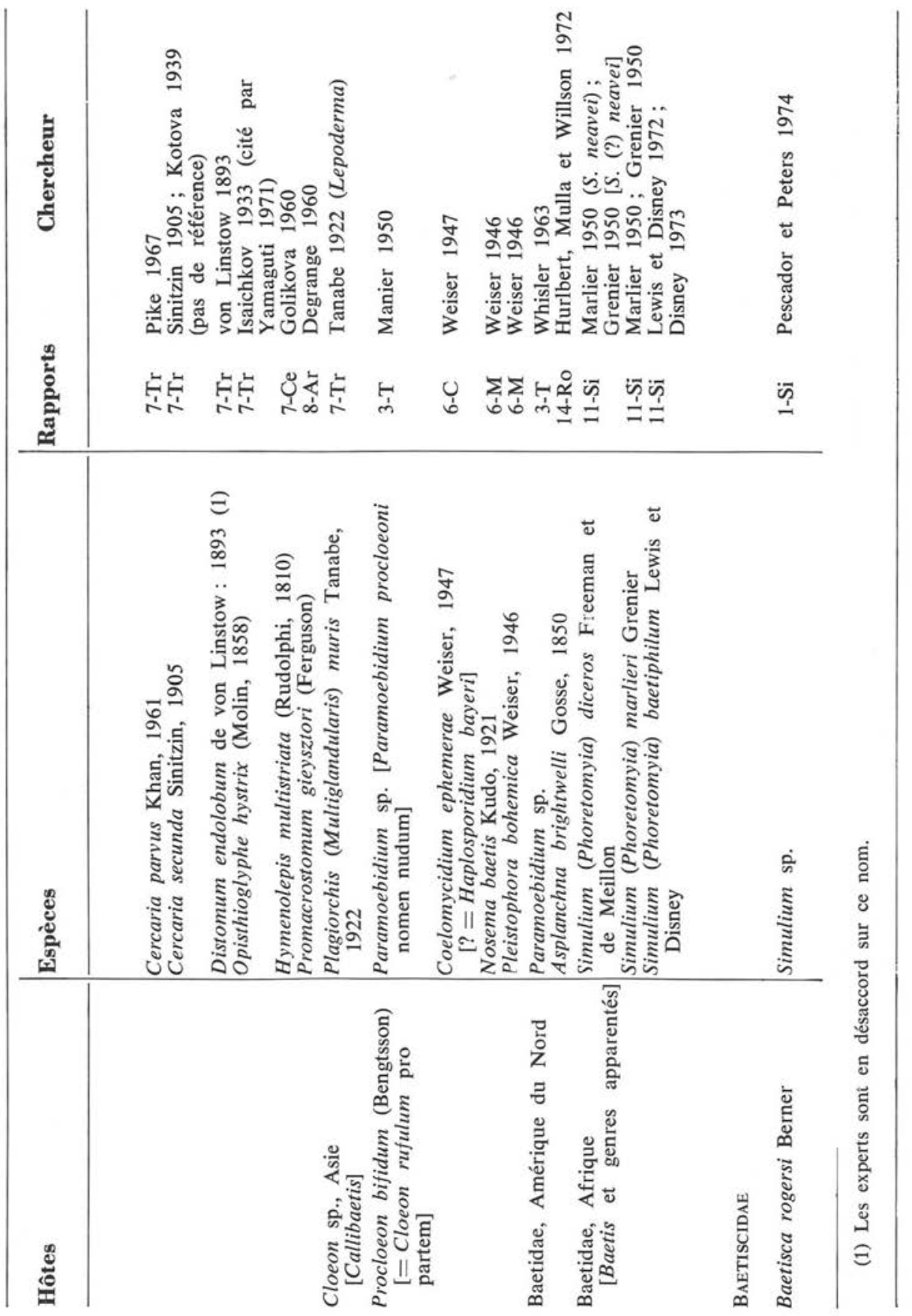




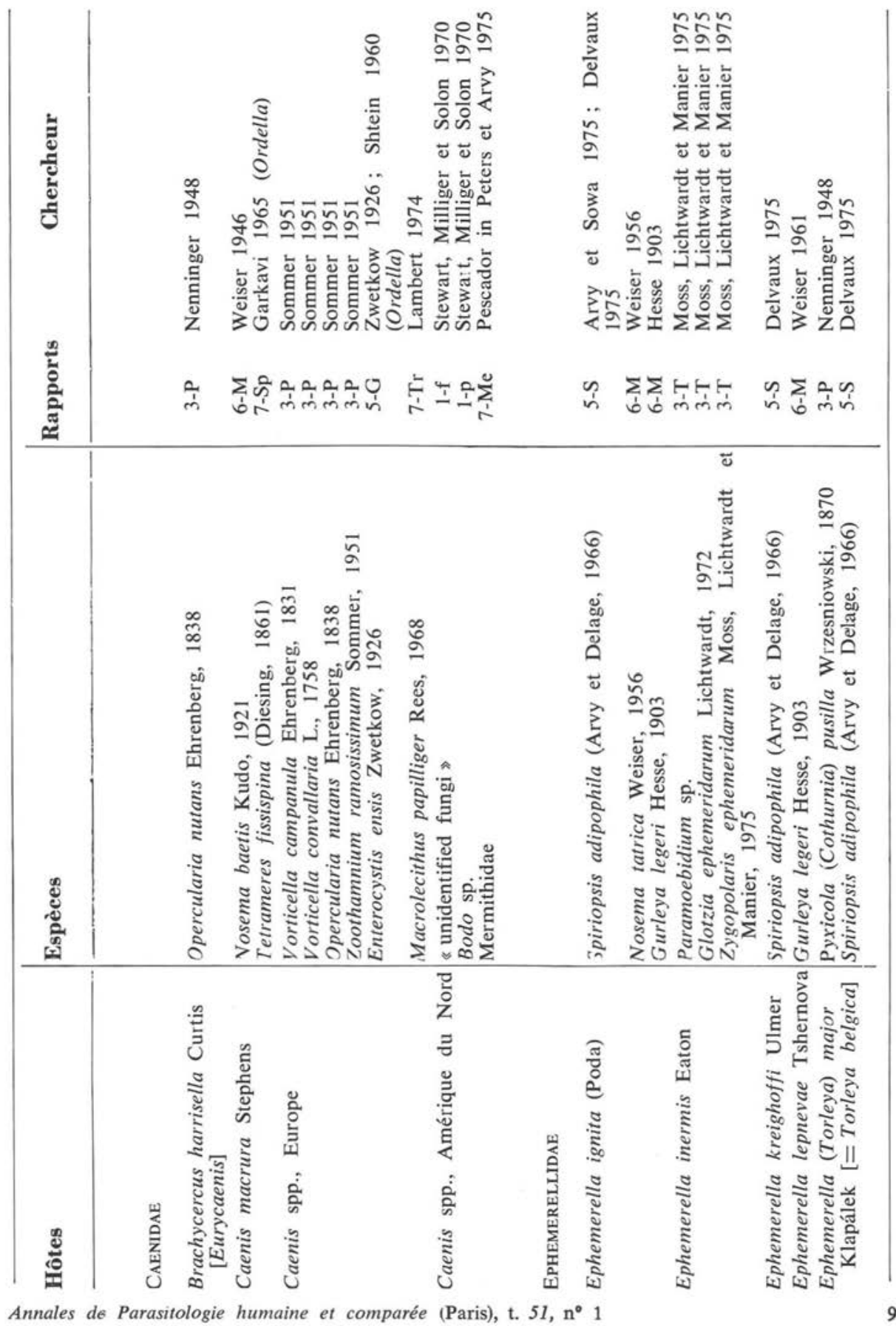




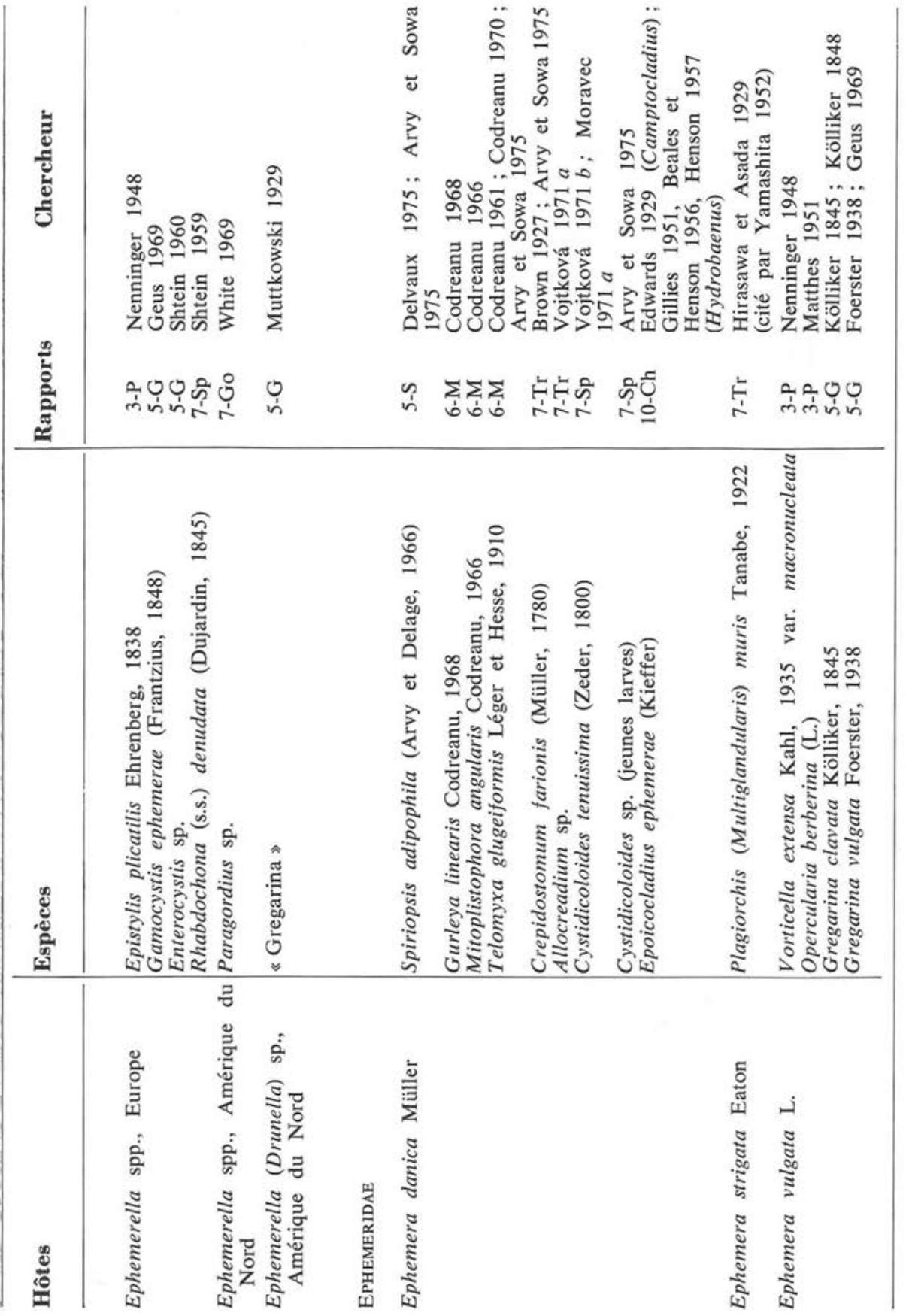




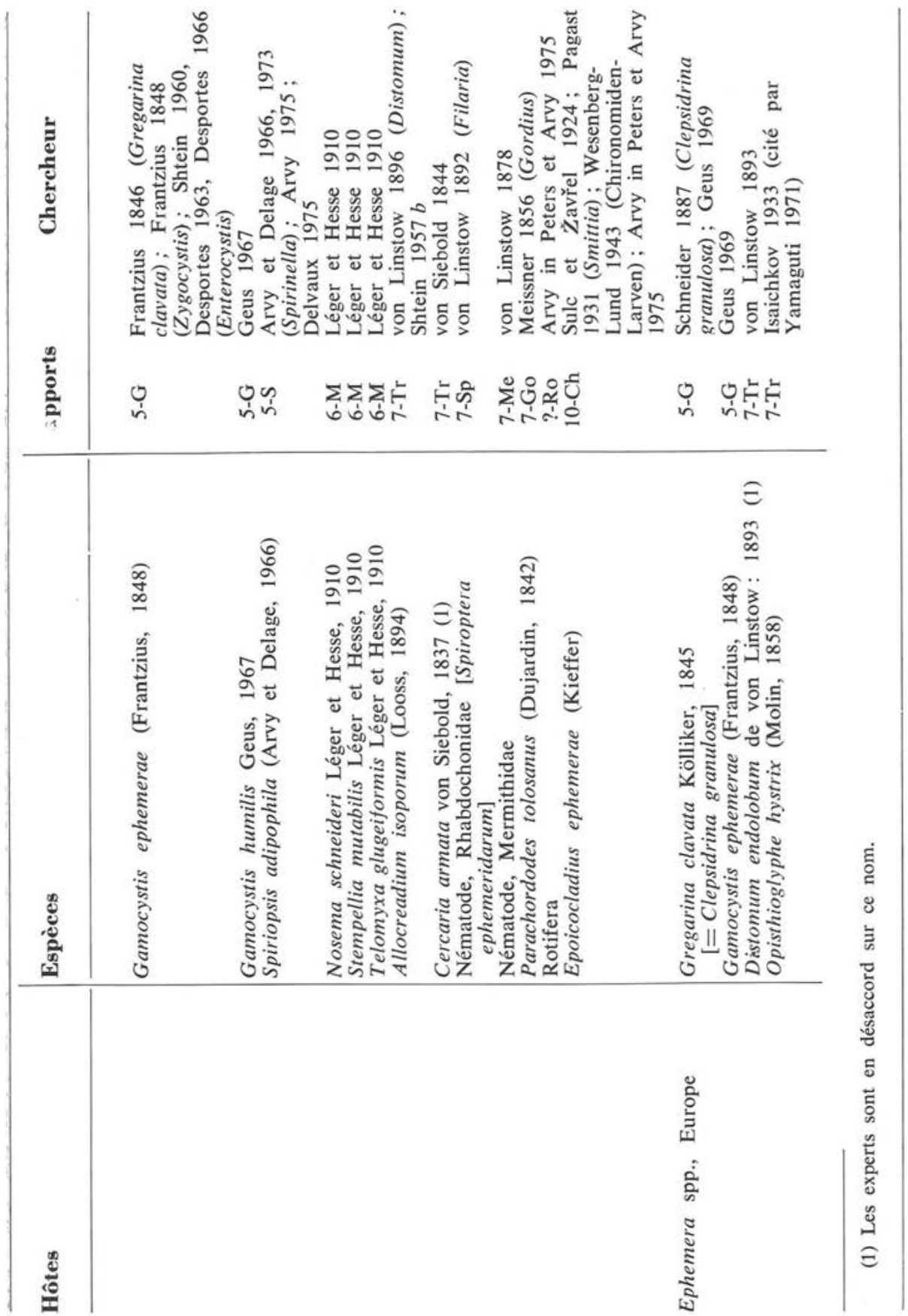




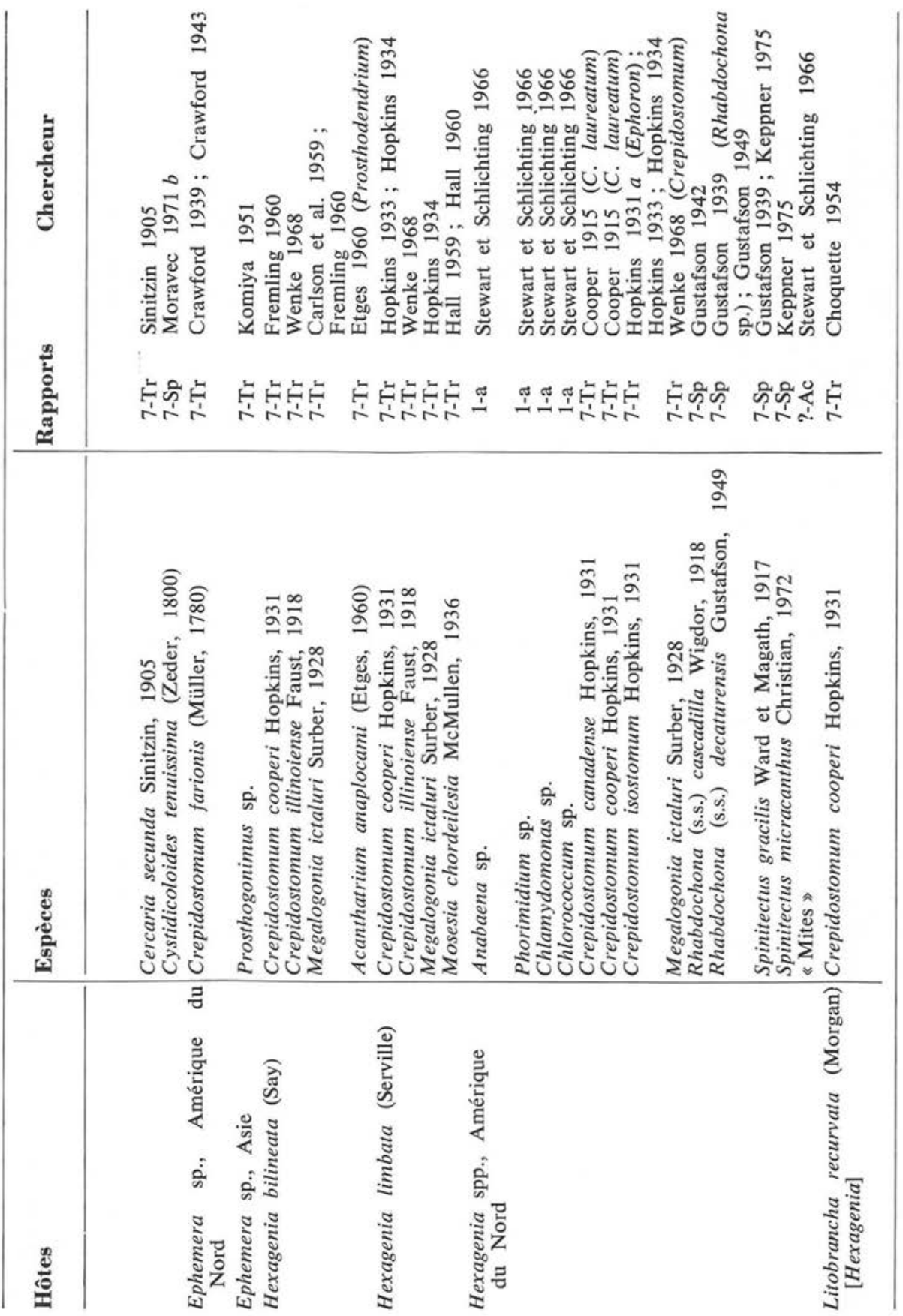




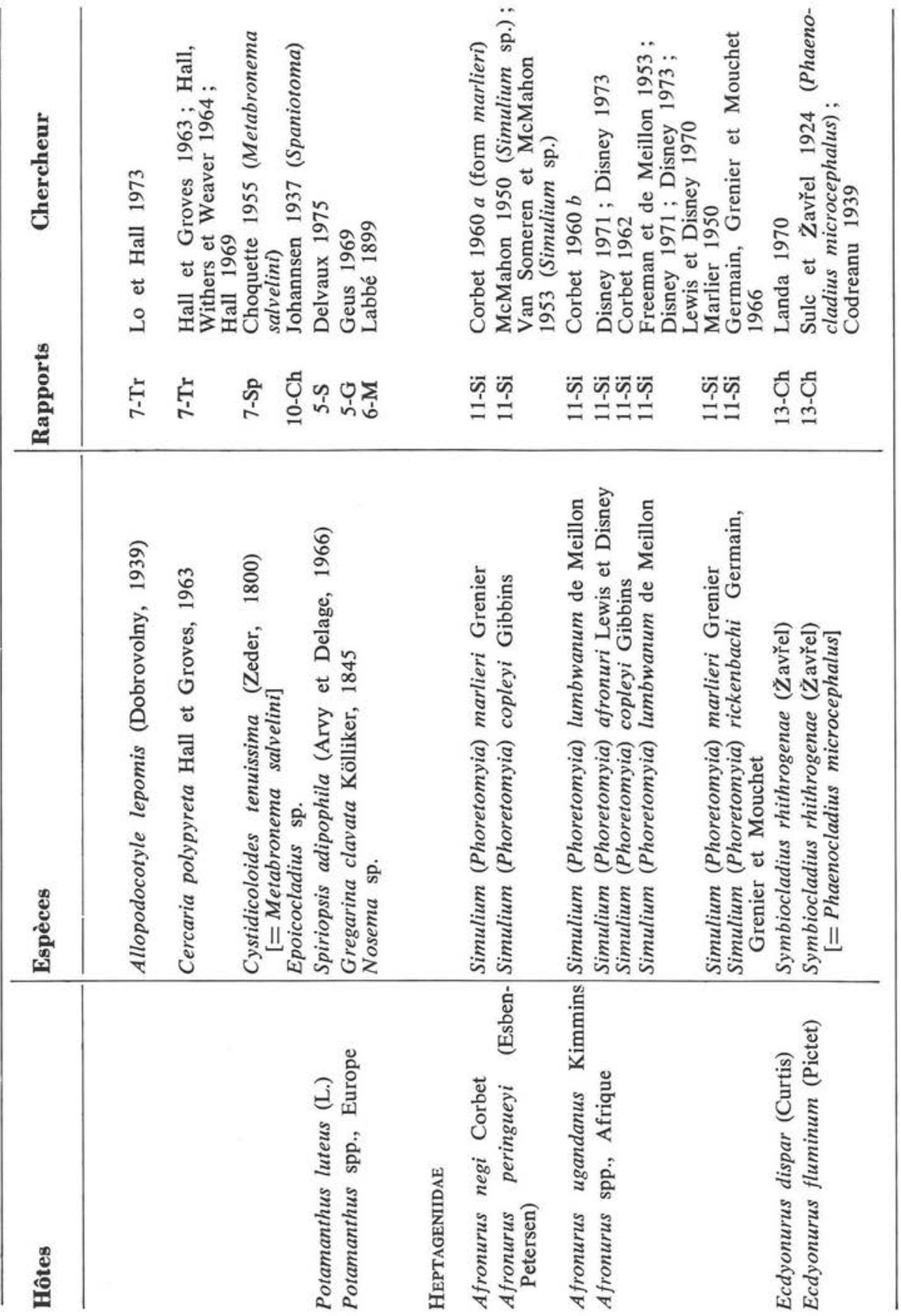




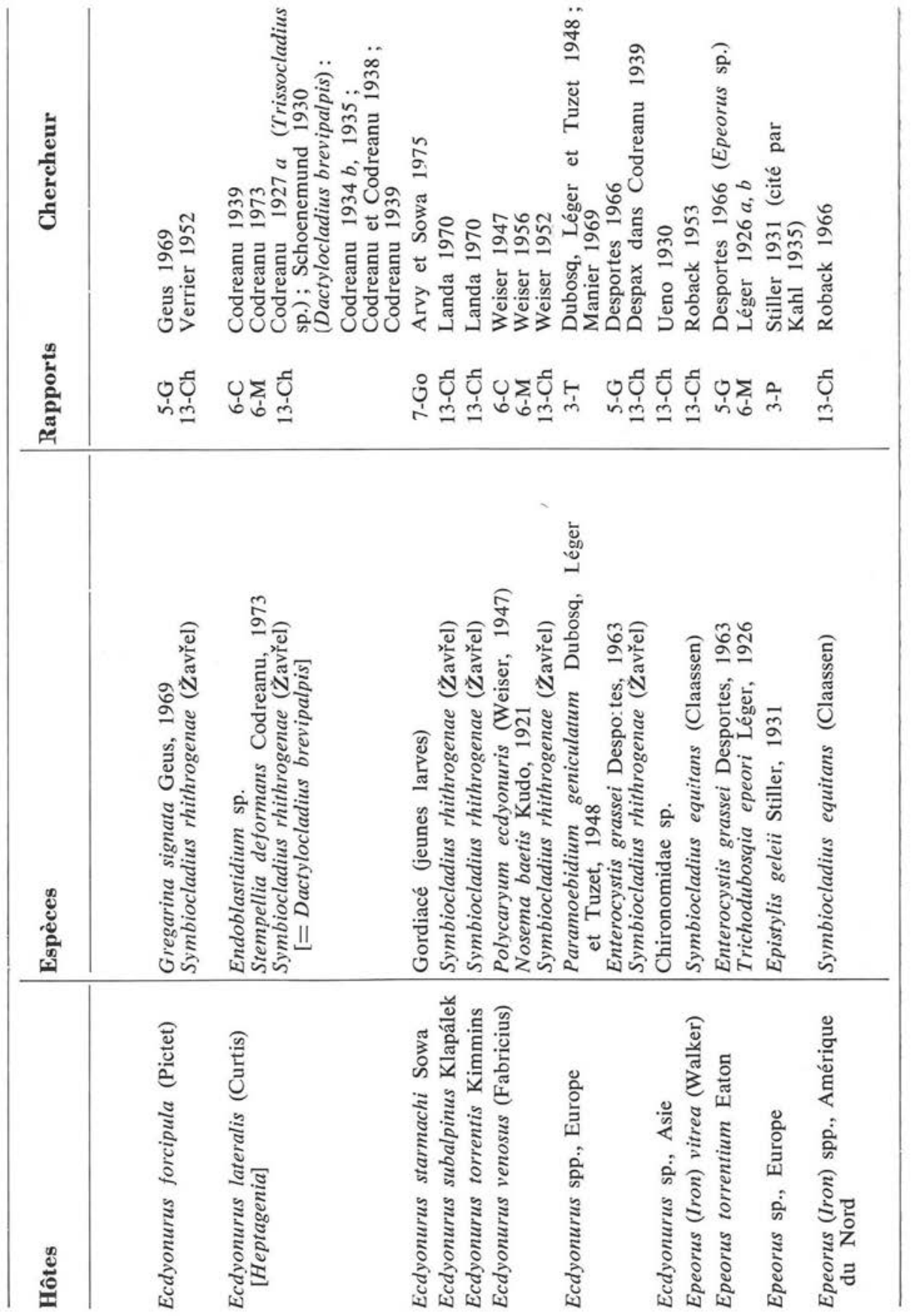


COMMENSAUX PARASITES D'EPHEMEROPTERES

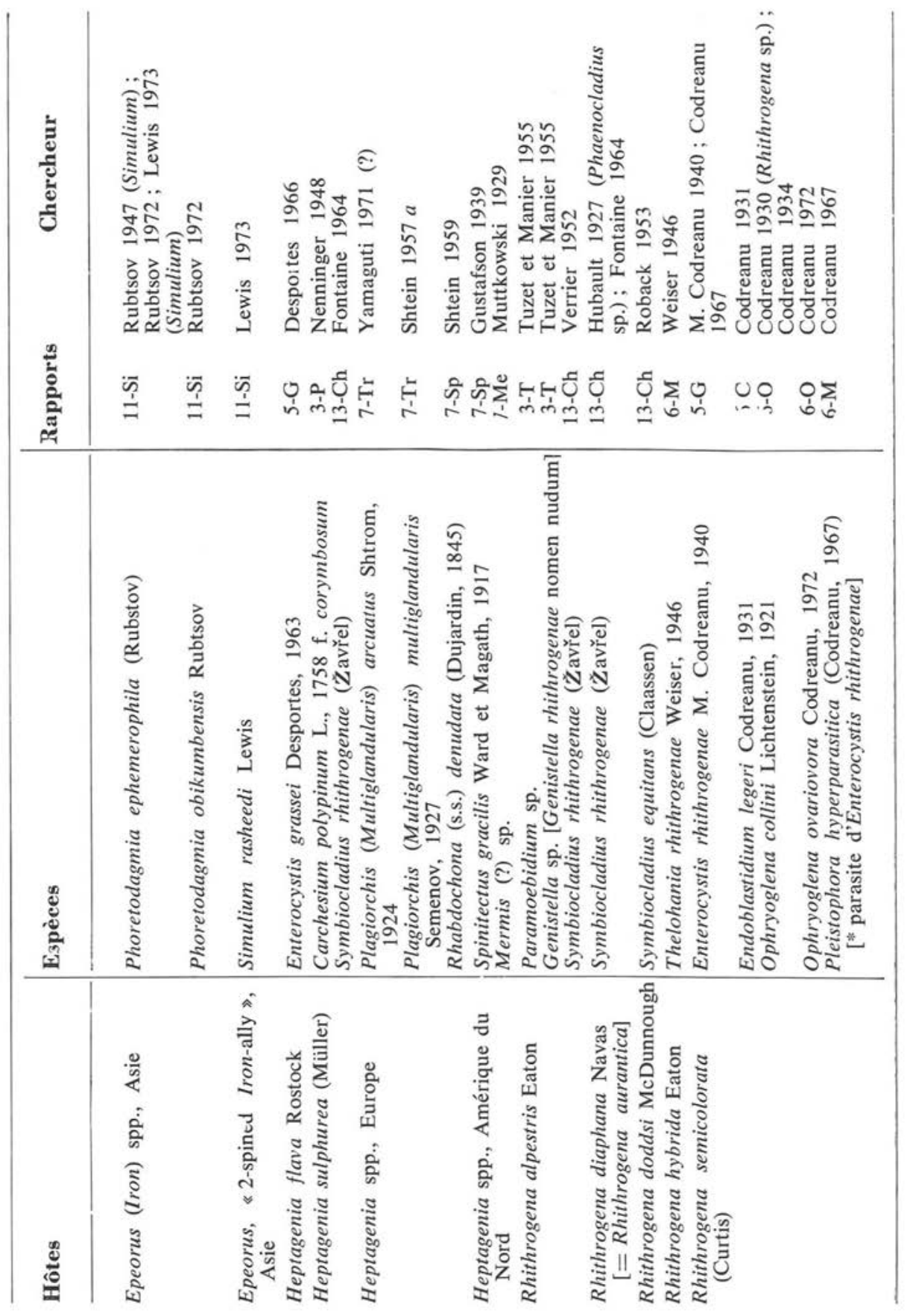




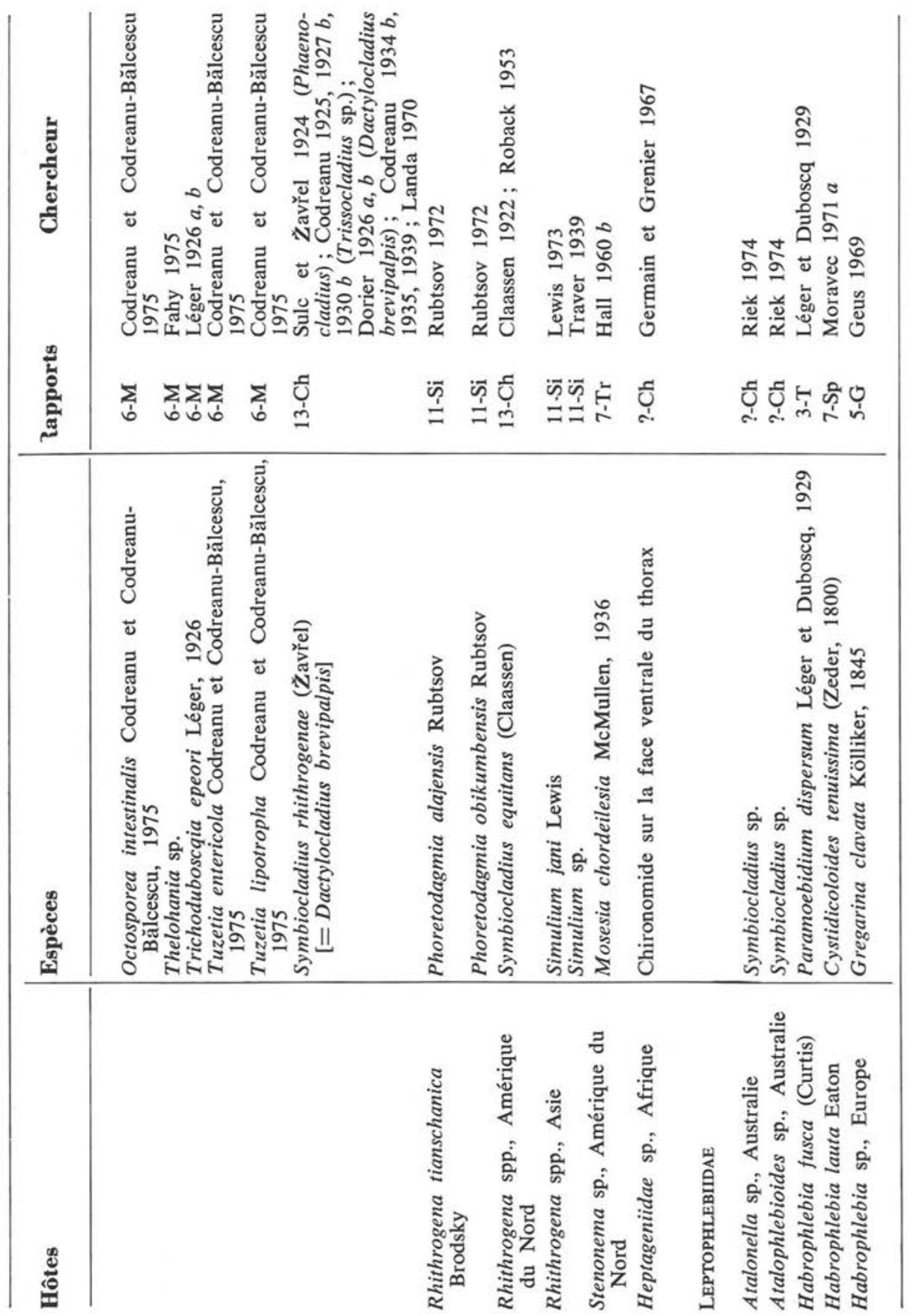




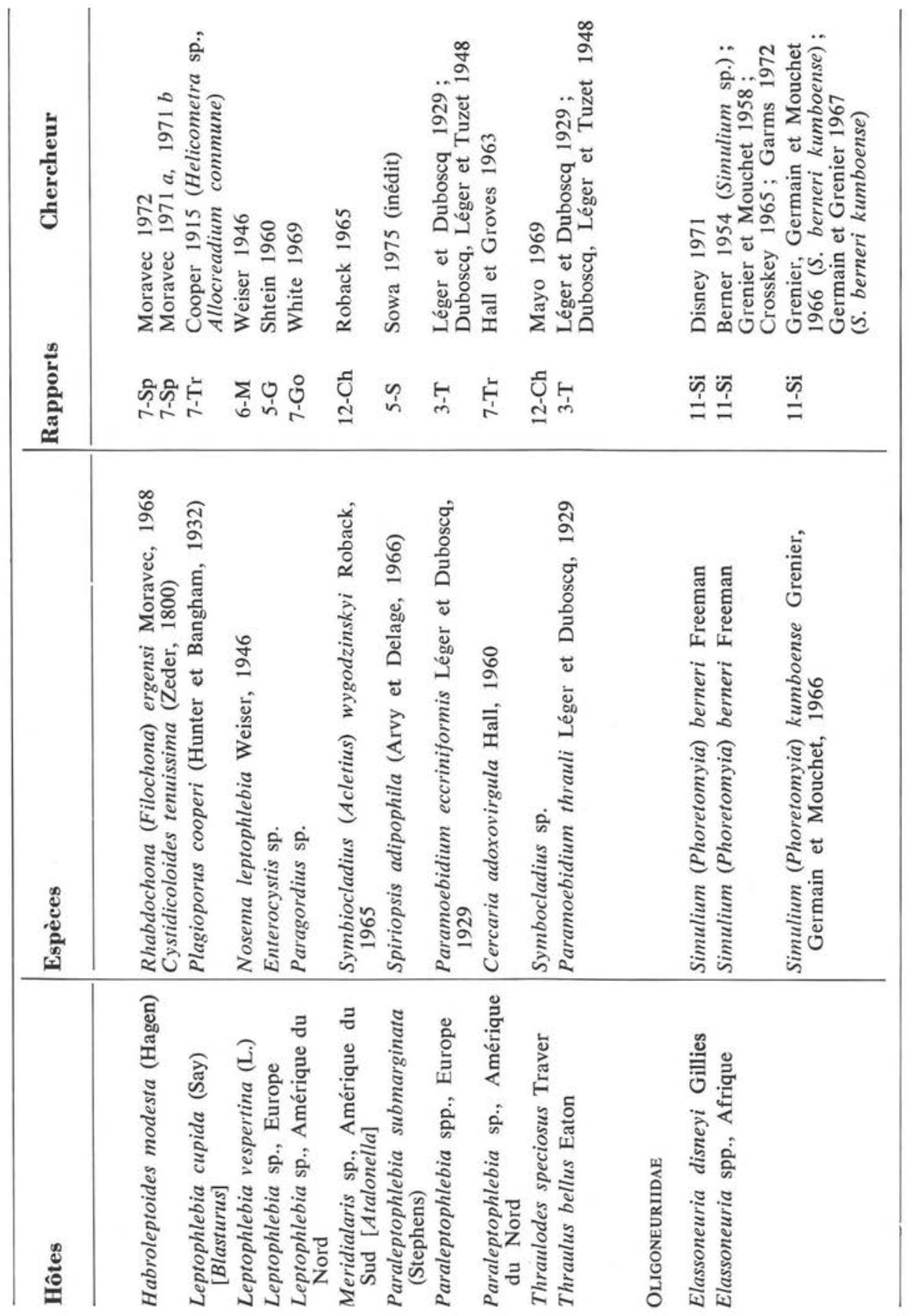




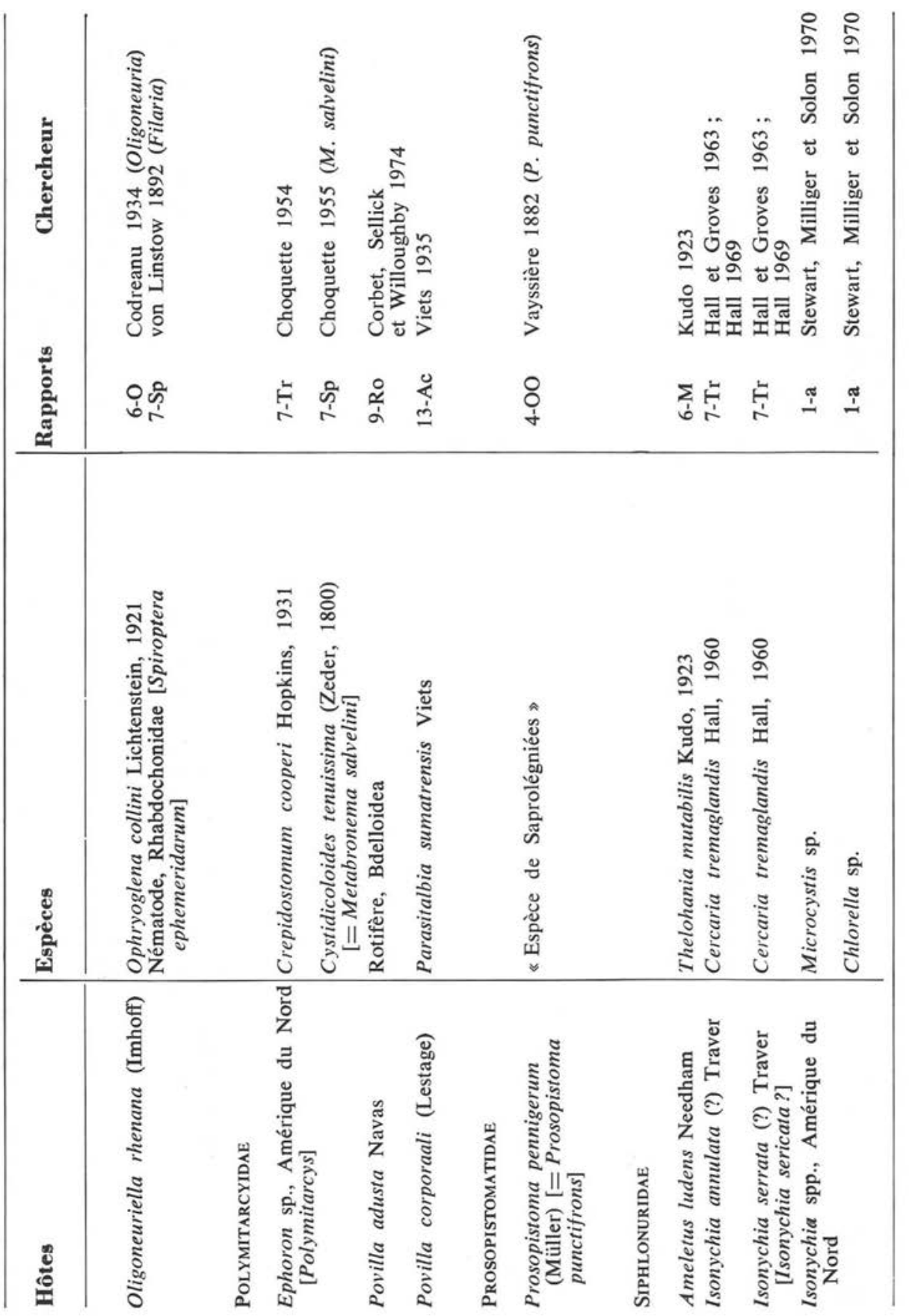




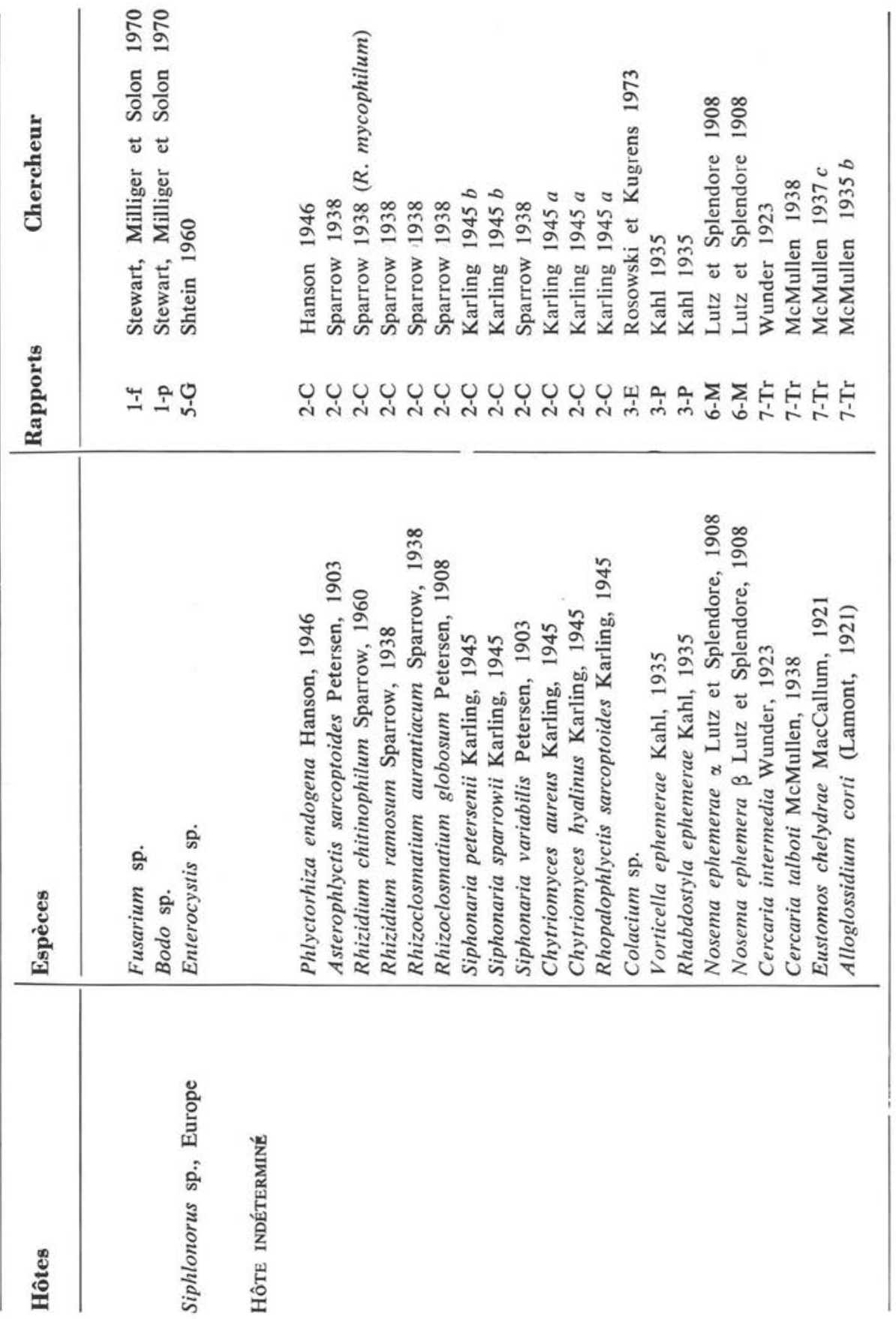




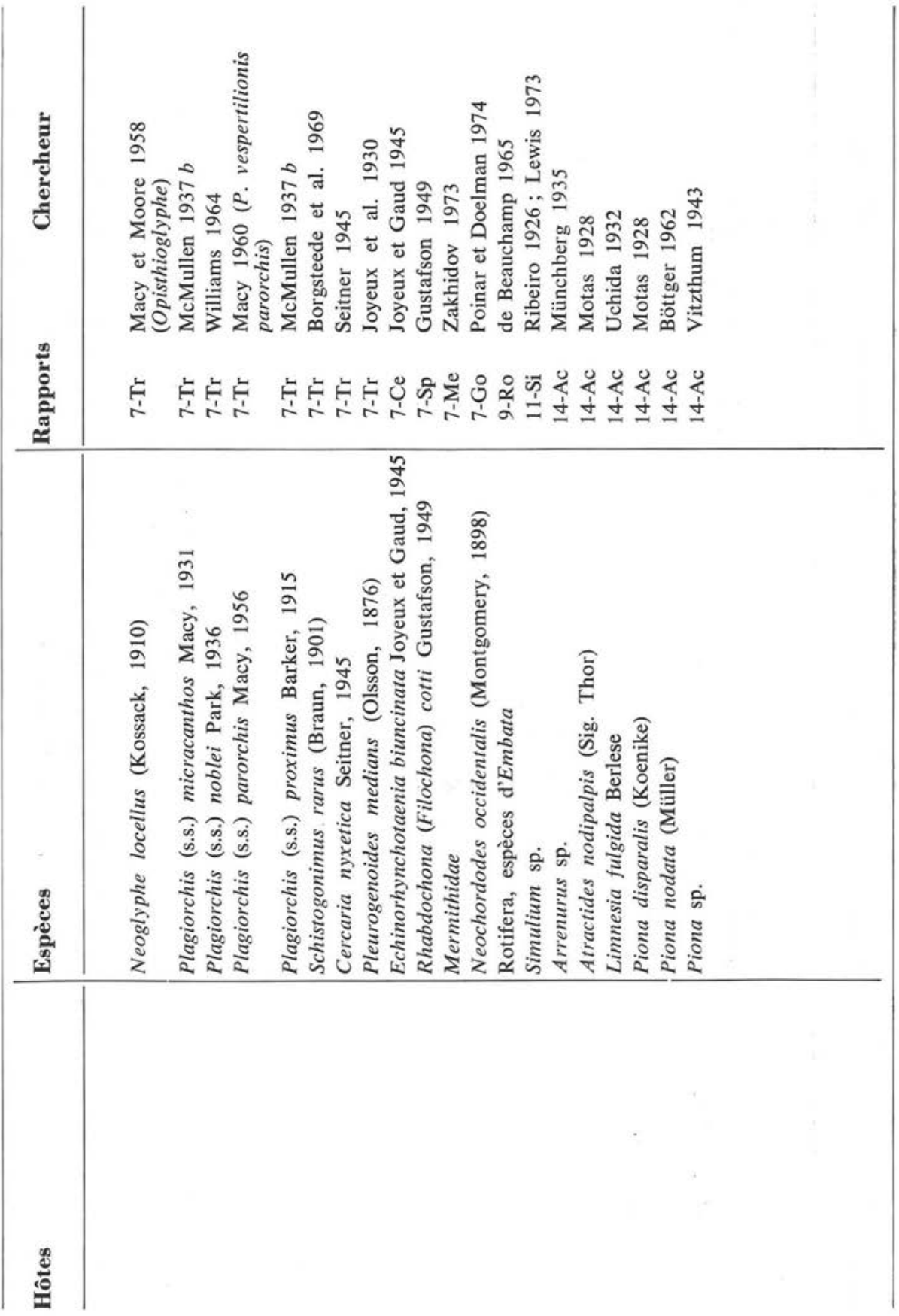




\section{Bibliographie}

1. Caullery (M.), 1950. - Parasitisme et symbiose. Douin édit., Paris, Encycl. Sci., Paris 1920.

2. Grassé (P.-P.), 1935. - Parasites et parasitisme. Colin, édit., Paris.

3. BAER (J.-G.), 1946. - Le parasitisme. Rouge, édit., Lausanne.

4. Henry (S.-M.), 1966. - Symbiosis, T. 1, pp. 9-11. Acad. Press. édit., Londres,

5. Delvaux (L.), 1975. - Spiriopsis adipophila (Arvy et Delage, 1966), parasite constant des populations d'Ephemera danica du Grand-Duché de Luxembourg, in: Sowa, Proc. $2^{e}$ Conf. Int. Ephemeroptera. Varsovie-Cracovie (à paraître). — La «Même'ck» des Pêcheurs du Grand-Ducité, Ephemera danica Müll. (Insecte éphéméroptère). Thèse de Biologie, Grand-Duché Luxembourg, 30 septembre 1975, 180 pages dactyl., 107 fig.

6. Demoulin (G.), 1955. - Un Elatéride prédateur d'Ephéméroptères. Bull. et Annales de Soc. R. Entomol. Belg., 91, 163.

7. Arvy (L.) et Peters (W.-L.), 1973. - Phorésies, biocoenoses et thanatocoenoses chez les Ephéméroptères, p. 254-312. In : Peters et Peters, Proc. $1^{\text {re }}$ Conf. Int. Ephemeroptera. E.-J. Brill, édit., Leyde, 312 p.

8. Peters (W.-L.) et Arvy (L.), 1975. - Phorésies, biocoenoses and thanatocoenoses in the Ephemeroptera. - Supplement, in: Sowa, Proc. $2^{\mathrm{e}}$ Conf. Int. Ephemeroptera. Varsovie-Cracovie. (à paraître).

9. Codreanu (R.) et Codreanu-Balcescu (D.), 1975. - Aperçu critique des parasites chez les Ephéméroptères, in: Sowa, Proc. $2^{e}$ Conf. Int. Ephemeroptera. Varsovie-Cracovie (à paraître).

10. RieK (E. F.), 1974. - Ephemeroptera (Mayflies), in : C.S.I.R.O., The Insects of Australia: Supplement 1974. Melbourne Univ. Press., Melbourne 1974, 33-34.

11. ARVy (L.), 1975. - Nouvelles données sur Spiriopsis adipophila (Arvy et Delage, 1966), Sporozoaire probable, in : Sowa : Proc. $2^{\mathrm{e}}$ Conf. Int. Ephemeroptera. Varsovie-Cracovie (à paraître).

12. ARvy (L.) et Sowa (R.), 1975. - Recherche sur les parasites internes des Ephéméroptères de la région de Cracovie. Ann. Parasit., hum. comp., 51, 117-126.

13. Chabaud (A.), 1976. $-\mathrm{N}^{\circ}$ 4. Keys to genera of the Spiruroidea, Habronematoidea and Acuarioides, in : Anderson, Chabaud and Willmott, CIH Keys to the Nematode Parasites of Vertebrates. Commonwealth Agricultural Bureau, Farnham Royal, Bucks, England 1976 (à paraître).

Il convient d'ajouter à nos observations celle de Britt (1962), suivant lequel l'examen d'environ un millier de larves d'Ephoron album et d'un millier de larves d'Ephemera simulans a révélé, dans $90 \%$ des cas, une infestation par des colonies d'un Péritriche: Carchesium sp. [Britt N. W.: Biology of two species of Lake Erie mayflies, Ephoron album (Say) and Ephemera simulans Walker, Bull. Ohio Biol. Surv., N.S., 1962, 1, (5), 1-70]. 